\title{
Nuclear Power Station Development History
}

\author{
Yuanyuan Liu \\ North China Electric Power University Baoding city, Hebei province, China \\ 2191385753@qq.com
}

Keywords: Nuclear power plant; Nuclear power; The development of history

\begin{abstract}
With the rapid development of economy, People's living standard is improving and people are also more and more high to the requirement of environmental protection. Now used in most countries is coal-fired power generation way, this way of electricity will be massive emissions of polluting substances into the air, for example: $\mathrm{SO}_{2}, \mathrm{NO}_{\mathrm{x}}$, which can destroy the self-regulation of nature and cause great damage to our living environment. Use of nuclear power is not only clean, but also, does not produce sulfur dioxide, carbon monoxide and other toxic gases, and the economy, save resources. From using nuclear power to now, the plant has experienced the change of generation after generation, continuously improving all kinds of equipment and the methods of combustion, to make nuclear power safer. This paper introduces the development history of nuclear power plant from set up to now and the development of nuclear power plant in the future expectations.

Energy is indispensable to the survival of humans upon earth and necessary conditions. The replacement of energy will cause the progress of human society. With the rapid development of social economy, the social productive forces in the continuously improve and human demand for energy is becoming more and more big. However, coal, oil and natural gas are non-renewable energy, and the earth reserves is limited. And the burning of fossil fuels may cause pollution to the environment, and influence the survival of the human environment. Nuclear energy has advantages of clean, low pollution and high thermal efficiency, and slowing down the temperature [1]. Use of nuclear power, $1 \mathrm{~kg}{ }^{235} \mathrm{U}$ all fission releases energy equivalent to 2700 tons of standard coal burning out of energy[2], which has a great development potential. Today, most countries in the world have dedicated to the study in the use of nuclear power. The table below for some countries in the use of nuclear power generation accounts for the total generating capacity of 2016.
\end{abstract}

Table 12016 nuclear power accounted for the proportion of total electricity generation

\begin{tabular}{lrr} 
country & annual energy output(Kilowatt hour ) & nuclear power proportion \\
America & 799 & $20 \%$ \\
French & 390 & $75 \%$ \\
Japan & 165 & $29 \%$ \\
Russia & 155 & $18 \%$ \\
Korea & 140 & $35 \%$ \\
Germay & 130 & $26 \%$ \\
Canada & 85 & $15 \%$ \\
Ukraine & 75 & $49 \%$ \\
China & 65 & $2 \%$ \\
England & 60 & $18 \%$ \\
\hline
\end{tabular}




\section{The Principle of Nuclear Power}

When there is external neutron bombardment of ${ }^{235} \mathrm{U}$ nucleus, nucleus absorbs a neutron splits into two smaller nuclei, and similar mass quality at the same time sent out two or three neutrons. The fission neutron again hits other ${ }^{235} \mathrm{U}$ nuclei, causing new fission. So continuous is called the fission chain reaction[3]. The chain reaction is shown below:

$$
{ }_{92}^{235} U+{ }_{0}^{1} n \rightarrow{ }_{37}^{93} R b+{ }_{55}^{141} C S+2_{0}^{1} n \text { (1) }
$$

Apparently, as long as the number of neutrons can control the strength of the chain reaction.Good at absorbing neutrons number of materials commonly used control rod position change to control the number of seeds in a chain reaction. In addition, the speed of the neutrons is very fast, so they must be slowed down with moderator before they can make it heavy nucleus fission [4]. The chain reaction produce a large amount of heat energy so with the circulating water to carry heat away to avoid core burnt due to overheating. Export of heat into the water of the secondary circuit of water vapor to push the turbine to do work, the mechanical energy into electrical energy, for the human production and life. Nuclear power plant reactor core reaction principle diagram as shown in the figure below

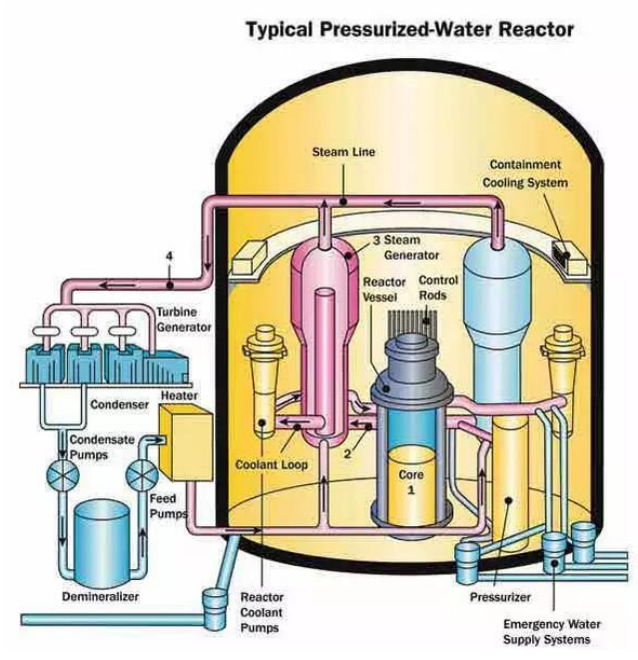

Figure 1. Principle

\section{The Early Stages of the Development of Nuclear Power Plant}

The former Soviet union in the ning, put the world's first experimental nuclear fission reactors in 1954, which marks the human first to use nuclear power applied to the construction of power generation of peace. Nuclear power plant in the early stage of development is to choose large graphite waste water reactor, graphite moderator, boiling water as a coolant. Light water goes through the core in the pressure tube and is heated to boil. And reactor building is just an ordinary factory workshop, at most only a "closed workshop" no doors, no containment. The reactor is pressure tube, which is by the pressure pipe under pressure[5].

Using graphite boiling water reactor design, there is a big security hidden danger that is the core melt explosion accident easily.

\section{Nowadays Development of Nuclear Power Plant}

Now each country choose according to the actual situation of its own national conditions to develop different nuclear fission reactor nuclear power plant, mainly light water reactor, heavy water reactor, 
graphite gas-cooled reactor pile and so on. These types of reactor are both using thermal neutron to bombard ${ }^{235} \mathrm{U}$ for the reaction.

Light Water Reactor Water. Nowadays the development of nuclear power plants are mostly the third generation of nuclear power unit, AP1000 and it gradually replaced the second generation nuclear power unit. AP1000 nuclear power unit actually did the melting of the core probability 5.08 $\mathrm{x} 10^{-7}$, which is reduced nearly 200 times [6].

The most commonly used reactor type is light water reactor in the nuclear power plant which is the use of light water as a coolant and moderato. According to the structure type can be divided into pressurized water reactor and boiling water reactor.

Pressurized Water Reactor. PWR is called the pressurized water reactor which is mainly composed of nuclear island, conventional island, control room, nuclear fuel plant, control room, and radioactive waste disposal, pump and so on a series of auxiliary workshop. PWR uses low enriched uranium for fuel. PWR is a second loop reactor. The task of the primary circuit is to transmit the fission heat as much as possible, and to ensure the fuel component not to burn. What s more, primary circuit is to use high temperature and high pressure water whose pressure is $12-16 \mathrm{MPa}$ and temperature is 300-330 degree centigrade. So we must put the core in a high pressure vessel which is called pressure shell[7].

Type Boiling Water Reactor. Boiling water reactor is the direct circulation, there is only one circuit. Cooling water in the reactor core is heated by addition of 285 degrees of boiling water vapor mixture, and then goes through the core upper steam separator, steam directly into the steam turbine driving steam turbine to do work. And boiling water reactor control rod is cross, from the bottom of the insert core, refueling without dismantling. It has fewer total shutdown, high load factor and high economic value. All installed in the containment are vessel reactor and extremely auxiliary system. Due to the boiling water reactor using negative pressure coefficient, flow control, core flow and flow control effect to the natural cycle of protection, etc., makes it a very safe nuclear power station [8]

Heavy Water Reactor. Heavy water reactor is given priority to pressure tube type heavy water reactor. Heavy water reactor uses $0.7 \%$ natural $235 \mathrm{U}$ for fuel pellets .It is a reactor secondary circuit. It uses high temperature and high pressure in a pile of heavy water $9.5 \mathrm{MPa}$ and 290 degrees of heavy water as a moderator. The coolant can use heavy water, also can use the light water, gas. The coolant can use heavy water, also can use the light water, gas. Coolant and moderator are separated, each has each loop, each has its own system, each in their own best working conditions. And heavy water reactor without shutdown and free loading and unloading of fuel, is easy to switch to another nuclear fuel.

Graphite Gas Cooled Reactor. Graphite gas-cooled reactor can be divided into natural uranium gas cooled reactor and high temperature gas cooled reactor. Graphite gas-cooled reactor has a circuit. Natural uranium gas cooled reactor is composed of 400 degrees Celsius, 2-3 $\mathrm{MPa} \mathrm{CO}_{2}$ as the coolant, with graphite as the moderator material and structure and its thermal efficiency is about $30 \%$.High temperature gas cooled reactor uses graphite as a moderator and 800-1300 degrees of helium as a coolant. Particle size of about $1 \mathrm{~mm} \mathrm{UO}_{2}$ fuel particle diffuses in the graphite substrate. High temperature gas cooled reactor core capacity is big, form a negative temperature coefficient of reactivity, alternative fuel, high thermal efficiency, good economy, the depth of the fuel consumption can be as high as $100000 \mathrm{mw} / \mathrm{ton}$. 


\section{Plant for the Future Development Trend}

Plant for the future development trend is the development of fast breeder reactor. Fast breeder reactor is a three circuits reactor. The first circuit is the core of coolant circulation system, with metallic sodium as a coolant. Sodium is also used as the heat transfer medium in secondary circuit. The first circuit of the heat transfers to the third circuit. The third circuit is a steam water system. And in a fast reactor, due to the use of fast neutrons, so there is no moderator. Fast breeder reactor not only can generate electricity, can also be generated in the process of power generation fuel and generate more fuel than the consumption of nuclear fuel. Reactions are as follows

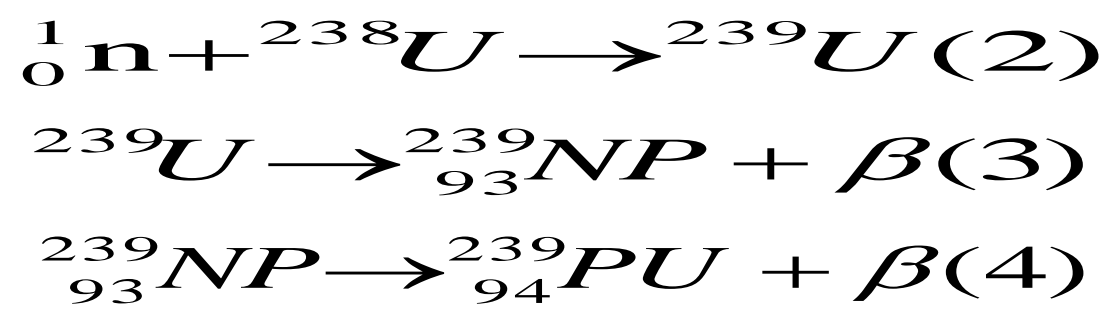

\section{The Development of Nuclear Power Plant Long-Term Goals}

Specific binding energy reflects the stability of the nucleus, the greater the specific binding energy, the greater the stability of the nucleus. According to the specific binding energy curves, the weight nuclear fission nuclear or lighter light fusion to the heavier nuclei is the process of exothermic reaction. At present, nuclear power plant is using heavier nuclear fission as lighter nuclei release of nuclear energy for power generation. The specific binding energy curve shows that fusion release more energy than nuclear fission , and nuclear fusion reactor does not produce greenhouse gases such as $\mathrm{CO} 2 . \mathrm{H}_{2}$ fuel can be extracted from the sea. This kind of fuel is inexhaustible. But fusion needs to use radioactive tritium, which are highly volatile heavy hydrogen escape. Thus radioactive pollution problems will happen. And after fusion, reactor vessel lining is bombard by neutron.Thus it has radioactive. Because this kind of fusion reactor components is under great load, and often must change [9]. The two problems restricting the development of nuclear fusion reactor, in the future development process, the two problems to solve, can we use nuclear fusion reactor.

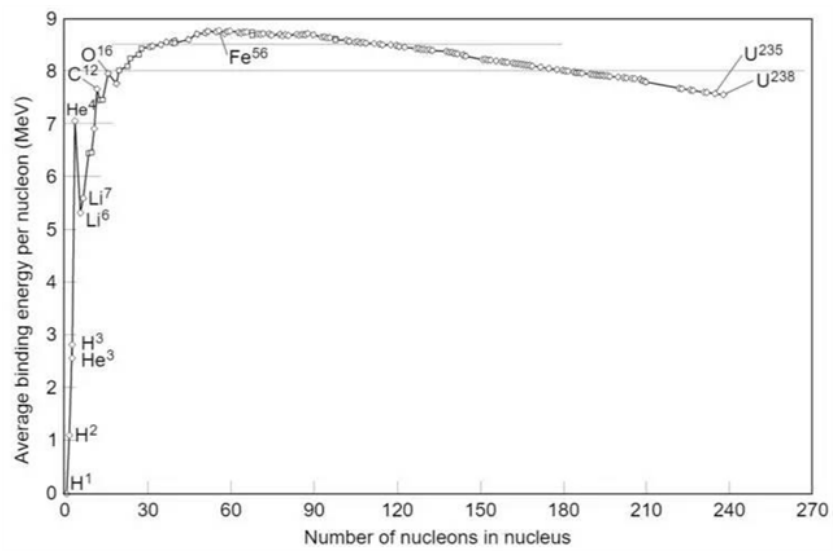

Figure 2. Principle

\section{Summary}

Since 1954, the world's first nuclear power plant was established in the former Soviet union, for the 
Soviet agricultural production projects provide the required power [10]. From when the first nuclear power plant set up till now, the plant has existed for more than 60 years. In the 60 years, the development of nuclear power plant experienced a generation after generation of improvement change, although some accidents happened, but did not prevent the people to the exploration of it. Believe in the future, people will develop more efficient and safer plants and benefit of all mankind.

\section{References}

[1] L.Shi. The British nuclear industry development research (1953-1987) [D]. Nanjing university, 2015

[2] http://guba.eastmoney.com/news,000519,588372973.html

[3] Z.H. Lin. The development and the application prospect of plant [J]. Nature, 2012, (02) : 63-68.

[4] Z.H. Lin. The development and the application prospect of plant [J]. Nature, 2012, (02) : 63-68.

[5] http://www.baidu.com/

[6] Y.Y.Ou. Improvement of nuclear power development and nuclear security in the world [J]. Journal of science and culture review, 2011, (5) : 17-22

[7] F.W.Tang. Xiangtian Wang and Jiakai Guo. Pressurized water reactor nuclear power plants First about the characteristics of the pressurized water reactor nuclear power plant [J]. Electric power technology, 1985, (7) : 69-73.

[8] X.M.He. Attaches great importance to the introduction of boiling water reactor nuclear power plants [J]. Energy, 1985, (3) : $44+35$

[9] The pros and cons of nuclear fusion reactor [J]. Foreign nuclear press, 1992, (5) : 31

[10]Q.Y. Zheng. The world's first set of plant stamps [N]. China stamp collecting news, 005 (005). 